\title{
Mortality in neurofibromatosis 1 : in North West England: an assessment of actuarial survival in a region of the UK since 1989
}

\author{
D Gareth R Evans ${ }^{\star, 1,2}$, Catherine O’Hara ${ }^{2}$, Anna Wilding ${ }^{1}$, Sarah L Ingham ${ }^{1}$, Elizabeth Howard ${ }^{1}$, \\ John Dawson ${ }^{1}$, Anthony Moran ${ }^{2}$, Vilka Scott-Kitching ${ }^{1}$, Felicity Holt ${ }^{1}$ and Susan M Huson ${ }^{1}$
}

\begin{abstract}
Neurofibromatosis 1 (NF1) is a comparatively common autosomal dominant disorder. However, relatively few studies have assessed lifetime risk; and information about the effect of NF1 on mortality remains uncertain. NF1 patients were identified using The North West regional family Genetic Register, which covers the 4.1 million people living in North West England, including the regions of Greater Manchester, Cheshire and Cumbria. Data relating to tumours and malignancies were obtained from The North West Cancer Intelligence Service. Death data for the general North West population were obtained from the Office of National Statistics. We identified 1186 individuals with NF1, of whom 1023 lived within the strict regional boundaries (constituting a region of North West England bound by The Pennines to the east and Irish Sea to the west, but excluding the conurbation of Liverpool (Merseyside) and the Wirral peninsula) and 131 had died. MPNST and glioma were found to be the two most common causes of reduced life expectancy among NF1 patients. In Kaplan-Meier analyses the median survival for NF1 patients was shown to be 71.5 years, with women living $\sim 7.4$ years longer than men. On average both men and women lived $\sim 8$ years less than their counterparts in the general population. Reduction in life expectancy for NF1 patients was found to be much lower ( 8 years) than the previously estimated 15-year decrease. Limitations relating to the underreporting of NF1 on death certificates were once again highlighted and should be considered in future investigations.
\end{abstract}

European Journal of Human Genetics (2011) 19, 1187-1191; doi:10.1038/ejhg.2011.113; published online 22 June 2011

Keywords: neurofibromatosis 1; mortality; autosomal dominant disorder

\section{INTRODUCTION}

A small number of studies have confirmed a generally shortened life expectancy in neurofibromatosis 1 (NF1) and NF2. Follow-up studies in NF1 have shown around a 15-year decrease in life expectancy in NF1 patients. ${ }^{1,2}$

There have been two previous NF1 cohort studies. Sorensen et al ${ }^{1}$ followed-up a nationwide cohort of 212 NF1-affected patients in Denmark. They obtained follow-up information on $99 \%$ over a 42 -year period. In a comparison with the general population, survival rates were significantly impaired in relatives with NF, worse in probands and worst in female probands. Malignant neoplasms or benign central nervous system tumours occurred in $45 \%$ of the probands, giving a relative risk of 4.0 (95\% CI, 2.8, 5.6). Compared with the general population, male relatives with NF had the same rate of neoplasms, whereas female relatives had a nearly twofold higher rate (relative risk, 1.9; 95\% CI, 1.1-3.1). Nervous system tumours were disproportionately represented.

Zoller et $a l^{2}$ conducted a 12-year follow-up study of 70 adult NF1 patients in the city of Göteborg, Sweden. Life expectancy, mortality and cause of death were investigated. The survival in the NF1 cohort was compared with that in the general Swedish population. Twentytwo deaths occurred in the NF1 group, whereas 5.1 deaths were expected in the general Swedish population $(P<0.001)$. The mean age at death was 61.6 years. Malignancy was found in $12(55 \%)$ of the deceased (soft tissue sarcomas in three and carcinomas in nine). Mean age at death was 15 years younger than expected in the general population.

A recent death certificate study from USA (1983-1997) established cause of death of $3770 \mathrm{NF} 1$ patients and again the mean age of death was around 15 years lower than in the general population ${ }^{3}$ with a PMR (proportionate mortality rate) of 34 for connective/soft tissue neoplasm, and a threefold increase in vascular disease for those $<30$ years. We have previously published on the incidence of the main soft tissue malignancy in NF1, malignant peripheral nerve sheath tumour (MPNST), from our NF1 register. ${ }^{4,5}$ This showed an annual incidence of 1.6 per 1000 individuals and an estimated $8-13 \%$ lifetime risk. ${ }^{4}$ Although there was evidence of a high death rate from MPNST, initial analysis of our NF1 register did not suggest a high death rate from cardiovascular disease. We therefore sought to identify the cause of death and life expectancy of NF1 individuals through the North West regional family Genetic Register.

\section{METHODS}

Patients

NF1 patients were identified from families referred to the genetic services in Manchester. The North West regional family Genetic Register service (GR)

\footnotetext{
${ }^{1}$ Genetic Medicine, The University of Manchester, Manchester Academic Health Science Centre, St Mary's Hospital, Central Manchester Hospitals Foundation Trust, Manchester, UK; ${ }^{2}$ North West Cancer Intelligence Service, NHS Foundation Trust, Manchester, UK

*Correspondence: Professor DGR Evans, Genetic Medicine, The University of Manchester, Manchester Academic Health Science Centre, St Mary's Hospital, Central Manchester Hospitals Foundation Trust, Manchester M13 9WL, UK. Tel: +44 (0) 161276 6206; Fax: +44 (0) 161276 6145; E-mail: gareth.evans@cmft.nhs.uk

Received 4 February 2011; revised 4 April 2011; accepted 4 May 2011; published online 22 June 2011
} 
covers a region of North West England based around Manchester with a population of 4.1 million and pro-actively obtains details of families with each condition. The region contains the relatively deprived urban area of greater Manchester (population 2.5 million) and relatively affluent rural areas. Information is sought on all affected and at risk blood relatives including date of birth, vital status, cancer status and address. The GR for NF1 was established in 1989. Affected individuals and their families are referred by general practitioners and specialists around the North West Region. Individuals are consented to the register recall system, which co-ordinates health screening and offers genetic testing at the appropriate age. At risk family members are encouraged to come to be evaluated. It also updates patients about new research and affected individuals also consent to be approached for research. The North West Cancer Intelligence Service (NWCIS) covers the same region of North West England. The NWCIS ascertains patients with malignancies of all sites, as well as benign CNS tumours, from hospital records, pathology records and death certificates. All hospitals are required to send pathology reports and discharge summaries on all malignant tumours and benign central nervous system tumours to the NWCIS.

Cases of NF1 were confirmed in the Clinical Genetics department using existing diagnostic criteria. ${ }^{6}$ Patients without confirmed NF1 such as those with multiple café au lait patches only were excluded. First-degree relatives of individuals with definite NF1 with clear reported neurofibromas and other NF1 features were assumed to have 'near definite NF1'. Date of birth of all affected family members was recorded on the register database and clinical databases for NF1. All deceased cases were confirmed where possible from medical records, cancer registry data or death certification. Confirmation of the vast majority of living affected cases was through clinic appointments, although some gave consent for their records to be assessed. Dates of last follow-up were either date of death or date the patient was last in contact with the department or other NHS service. For all those with no recent clinical follow-up, vital status was confirmed through the NHS tracing system. All cases were also checked against the NWCIS for cancer and vital status. NF1 cases were divided into group 1: individuals within the strict regional boundaries and group 2: those residing outside the boundaries. Kaplan-Meier curves were derived from date of birth to assess actuarial survival to death. To avoid any ascertainment bias only those residing within the regional boundaries (group 1) were used for the latter analysis.

Proportionate mortality ratios were calculated as the number of observed deaths from a specified condition in the NF1 population divided by the number of deaths expected from this condition in the general population. The expected number of deaths associated with a particular condition was calculated from death registration data for North West England obtained from the Office for National Statistics. ${ }^{7}$

\section{RESULTS}

A total of 1186 individuals from 644 families with a confirmed or near-certain diagnosis of NF1 were identified of which 131 (11\%) had died between 1957 and 2009. The birth years of all NF1 patients are presented in Table 1 . The median year of death was 1998 with all but 25 (19\%) deaths occurring after the inception of the NF1 register. Cause of death from death certification was established in 130/131 (99\%) cases (Table 2). Of those living within the strict regional boundaries (group 1) 109/1023 (10\%) had died. The most common cause of death was MPNST with 34/131 (26\%) of deaths being due to this condition (Table 2). In comparison 6/19 (32\%) of non-regional deaths (group 2) were due to MPNST and 28/111 (25\%) of regional (group 1) deaths.

Glioma was the second most common cause of death and the most common cause in those $<20$ years of age (Table 2). Conversely, cardiovascular deaths were not particularly common with only $10 / 80$ deaths in those aged $<50$ years being attributed to this cause, and only six of these deaths having a clear vascular origin. However, 8/10 cardiovascular deaths $<50$ and $19 / 26(73 \%)$ of all cardiovascular deaths occurred in male NF1 cases. Two deaths aged 34 and 44 years were due to blood vessel disease secondary to radiotherapy in
Table 1 Years of birth of 1186 NF1 patients in groups 1 and 2

\begin{tabular}{lccc}
\hline Year of birth & All patients & Group 1 & Group 2 \\
\hline $1900-1909$ & 4 & 3 & 1 \\
$1910-1919$ & 5 & 5 & 0 \\
$1920-1929$ & 19 & 16 & 3 \\
$1930-1939$ & 32 & 28 & 4 \\
$1940-1949$ & 69 & 62 & 7 \\
$1950-1959$ & 143 & 125 & 18 \\
$1960-1969$ & 181 & 159 & 22 \\
$1970-1979$ & 204 & 172 & 32 \\
$1980-1989$ & 231 & 199 & 32 \\
$1990-1999$ & 213 & 184 & 29 \\
$2000+$ & 85 & 70 & 15 \\
Total & 1186 & 1023 & 163 \\
\hline
\end{tabular}

childhood. The clearest indication of a vascular death unrelated to radiotherapy was a ruptured thoracic aneurysm in a 20 -year-old male. The mean and median age at death in the regional cohort from all causes was 43.55 and 44.13 years of age. There were two deaths related to bleeding into a plexiform tumour post-surgery aged 40 and 45 years that were registered as tumour-related deaths but could have had a part vascular origin. There were nine deaths that are likely to have been related to radiotherapy for childhood optic glioma. Two vascular deaths aged 32 and 44 years in males, four MPNSTs occurring 16-25 years after radiotherapy and three high-grade gliomas. Four of the six glioma deaths $<20$ years of age were due to more aggressive than usual optic gliomas in persons aged 1.5, 3.4, 3.9 and 14.5 years. None had received radiotherapy.

\section{Kaplan-Meier analysis}

Using the strict regional dataset of 1023 individuals, median survival was 71.5 years with $50 \%$ of NF1 patients dead by 71 years of age. Women with NF1 lived for $\sim 7.4$ years longer than men although this did not quite reach statistical significance (Figure $1 ; P=0.07$ ). This was largely because the curves did not separate until after 55 years of age. Tumour deaths, apart from female breast cancer $(P=0.013)$, did not vary significantly between men $(32 / 62,52 \%)$ and women $(42 / 68,62 \%)$ $(P=0.355)$. In the enlarged dataset deaths due to MPNST were also similar between the sexes (18:16 for women and men, respectively), conversely there were nine female glioma deaths compared with only four in males although this was not significant $(P=0.3626)$, in the strict regional population the ratio was 8:2.

\section{Expected regional deaths}

Expected deaths for the general North West population is shown in Figures 2 and 3 . There was a loss of 8 years of life expectancy for both NF1 males and females $(P<0.01)$ (Table 3$)$.

In a population of 1000 from the local regional population we would expect $60(6 \%)$ to have died by 50 years of age as opposed to the $19 \%$ for both males and females in the NF1 population. ${ }^{7}$ In all $0.9 \%$ of males and $1.1 \%$ of females in the UK will have died from tumour related causes by 50 years of age using current death rates. ${ }^{7}$ By 50 years of age $0.1 \%$ of males and $0.06 \%$ of females will have died from a malignant brain tumour and a further $0.3 \%$ of females will have died of breast cancer. Furthermore, $0.9 \%$ of males and $0.35 \%$ of females will have died by 50 years of age from cardiovascular causes. Comparing the NF1 associated deaths against deaths within the local 
Table 2 Cause of death by age cohort in NF1 patients

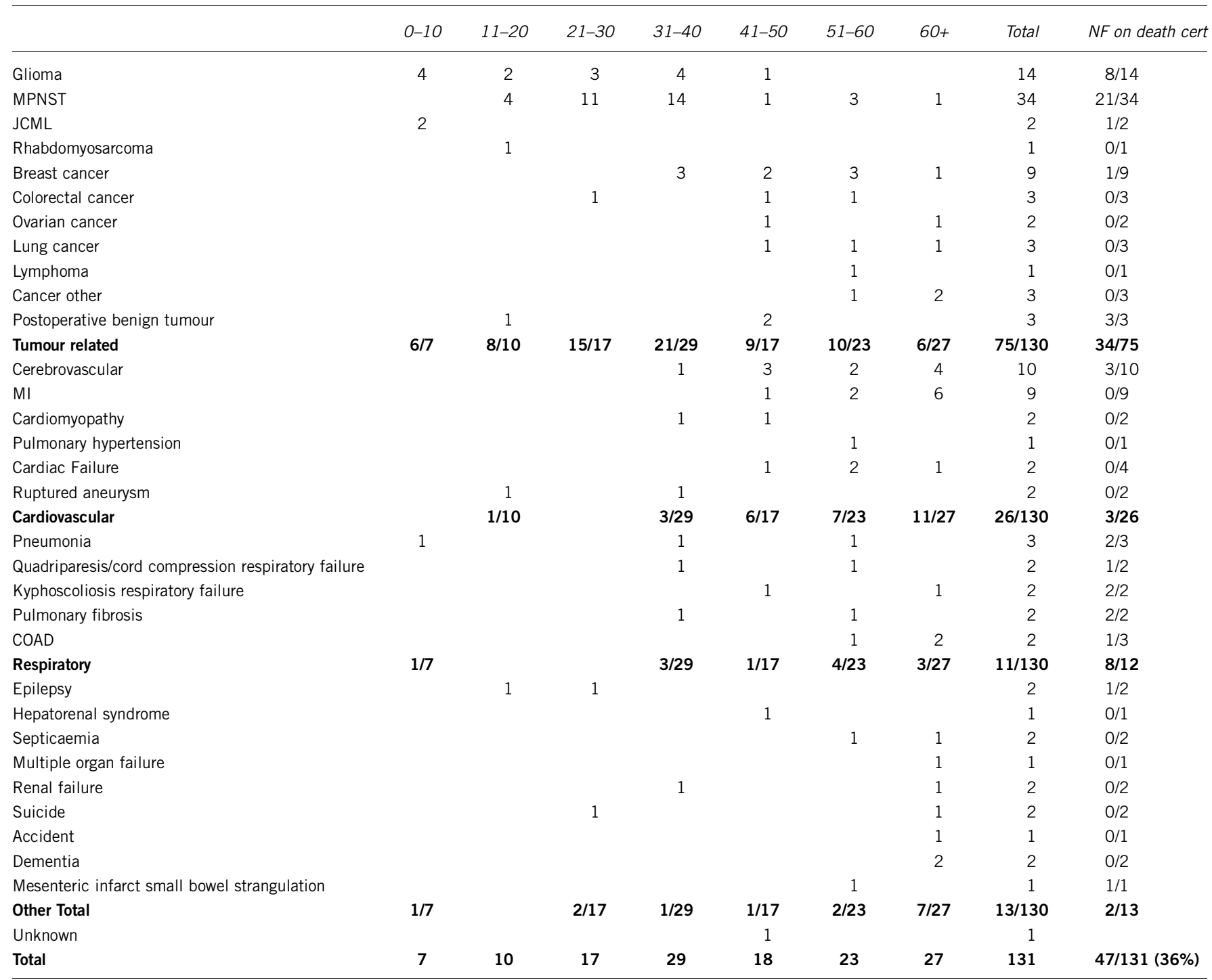

Values in bold refer to the total deaths in each disease category by age.

North West population, cardiovascular disease was reported four times more frequently in NF1 males ( $\mathrm{PMR}=4.1 ; 95 \% \mathrm{CI}, 1.4-2.6$ ) than the general population. Similarly breast cancer death was reported 3.5 times more frequently in NF1 females ( $\mathrm{PMR}=3.5 ; 95 \%$ CI, 1.3-7.7). Brain tumours were also reported more often in NF1 females than the local population (Table 4) but not statistically significantly so in males ( $\mathrm{PMR}=6.7 ; 95 \% \mathrm{CI}, 0.8-24.1$ ).

The most notable result was that of death attributable to MPNST. MPNST was reported as the cause of death significantly more often than expected in both NF1 males and NF1 females ((males $\mathrm{PMR}=3819.6$; 95\% CI, 1971.4-6672.5), (females $\mathrm{PMR}=7788.2 ; 95 \%$ CI, 4355.7-12 846.2)).

\section{DISCUSSION}

Our analysis of mortality in NF1 has again shown a reduction in life expectancy, but we found 50\% of NF1 affected individuals can expect to live beyond 71 years of age. The main causes of early death were MPNST and glioma, as expected from previous investigation. However, we did not replicate the findings of Rasmussen et al ${ }^{3}$ with only
10 vascular deaths recorded by 50 years of age. Nonetheless cardiovascular deaths were still four times more common in males with NF1 than expected overall. In the local North West population we would expect $6 \%$ of the population to have died by 50 years of age of which 9.2 males and 11 females (in 1000 individuals) would have died from tumour-related causes. In our population of just over 1000 there were 19 male cancer deaths and 29 female deaths with many individuals yet to attain 50 years of age.

Malignancy risk in NF1 outside MPNST is probably not substantially increased in common tumours apart from gliomas, which are usually low grade and not considered malignant. Nonetheless this study found $8 \mathrm{NF} 1$ patients died from glioma disease. Of note is that six of these deaths occurred before 20 years of age.

Our study differs from the previous two cohort studies ${ }^{1,2}$ in that it ascertained all individuals with NF1 rather than just adults. The Swedish cohort study in particular was based on a cohort first described by Samuelsson who had to be $>20$ years of age in $1978 .^{8}$ As such the population still remains quite young and most deaths are still yet to occur. Deaths are therefore biased to a younger age where 


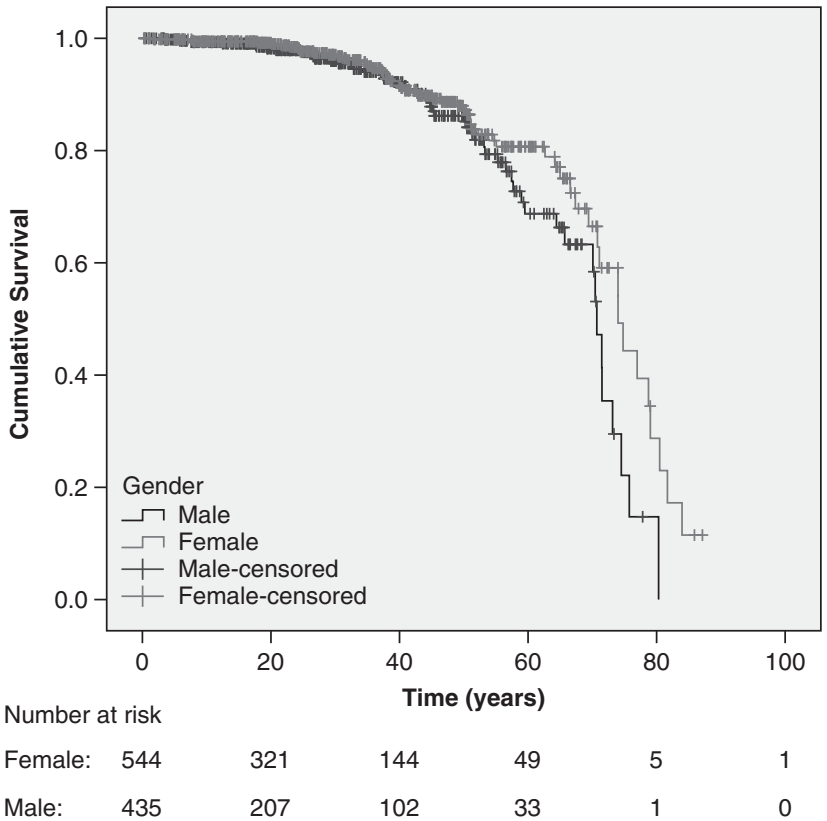

Figure 1 Actuarial survival in all individuals with NF1 showing separate female and male gender in years.

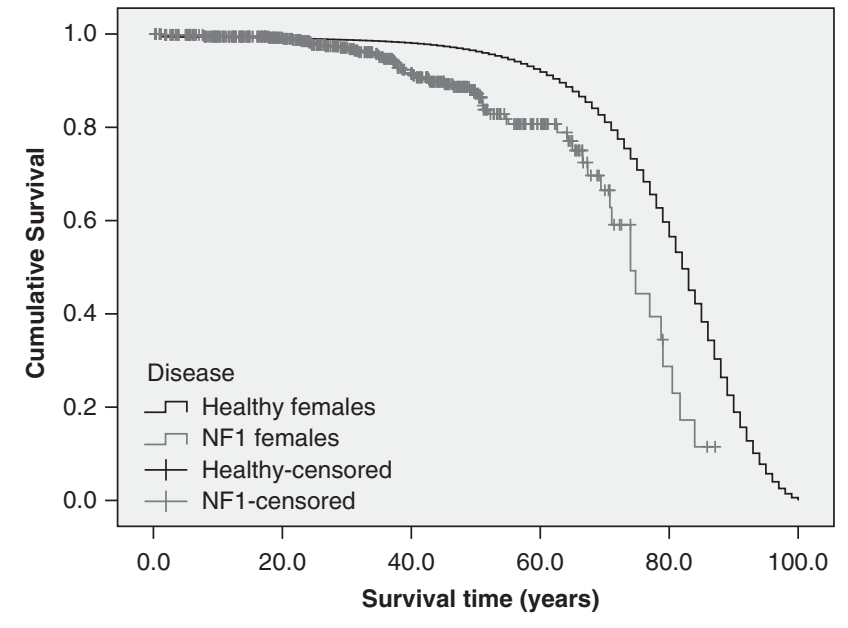

Figure 2 Actuarial survival in females with NF1 compared with females in the general population.

MPNST and glioma predominate. This is reflected in 26\% (34/131) of deaths even in the unbiased regional cohort being due to MPNST when only $8-13 \%$ of patients would be expected to develop this complication in their lifetime. ${ }^{4}$ Nonetheless, our Kaplan-Meier analysis reduces the bias as living individuals are included. The median survival is substantially higher than the mean and median ages at death at 71.5 years. This would suggest that despite ascertaining a younger NF1 population NF1 individuals are living closer to the population norm than estimated from the previous cohort studies.

Although a death certificate study might be expected to overcome the ascertainment bias of cohort studies they are dependant on NF1 being accurately recorded on the death certificate. In our study only $36 \%$ of NF1 patients on whom we were able to obtain details of death certification had NF1 as a contributing cause on the death certificate.

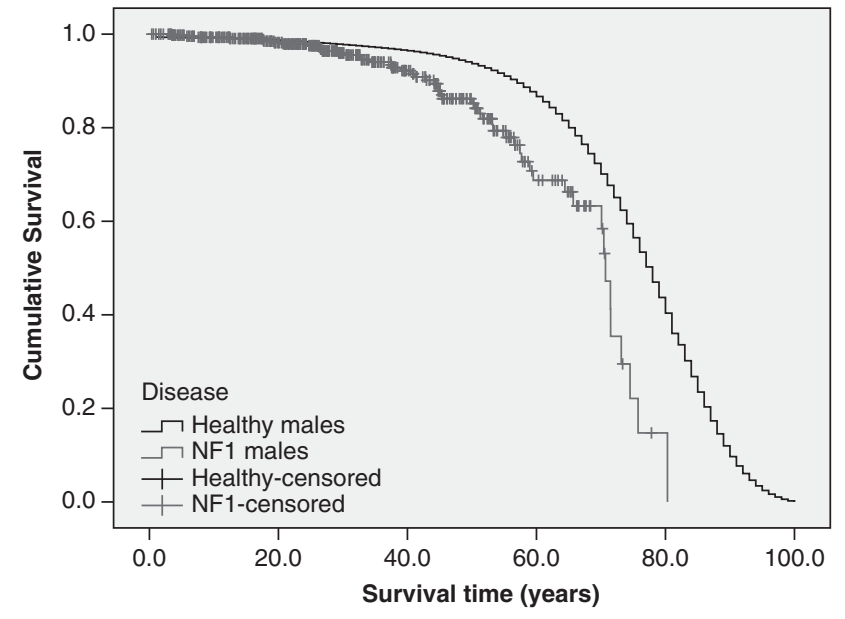

Figure 3 Actuarial survival in males with NF1 compared with males in the general population.

Table 3 Survival in NF1 males and females compared with the general population

\begin{tabular}{llccc}
\hline & & \multicolumn{3}{c}{ Median } \\
\multirow{2}{*}{ Gender } & Disease & Estimate & Lower & Upper \\
\hline \multirow{2}{*}{ Male } & Healthy & $\mathbf{7 8 . 0}$ & 77.7 & 78.3 \\
& NF1 & 70.7 & 69.2 & $72.2(P<0.001)$ \\
\multirow{2}{*}{ Female } & Healthy & $\mathbf{8 2 . 0}$ & 81.7 & 82.3 \\
& NF1 & $\mathbf{7 4 . 0}$ & 69.7 & $78.3(P<0.01)$ \\
\hline
\end{tabular}

Values in bold refer to the estimated years of survival.

Table 4 The PMR for deaths of individuals with NF1 for selected medical conditions

\section{No. of cases}

\begin{tabular}{|c|c|c|c|c|c|}
\hline codes & Condition & Gender & Observed & Expected & PMR (95\% Cl) \\
\hline \multirow[t]{2}{*}{ 100-199 } & Cardiovascular: & Male & 19 & 4.7 & $4.1(2.4,2.6)$ \\
\hline & & Female & 7 & 4.1 & $1.7(0.7,3.5)$ \\
\hline C50 & Breast cancer & Female & 6 & 1.7 & $3.5(1.3,7.7)$ \\
\hline \multirow[t]{2}{*}{ C71 } & Brain tumour & Male & 2 & 0.30 & $6.7(0.8,24.1)$ \\
\hline & & Female & 8 & 0.27 & $29.5(12.7,58.1)$ \\
\hline \multirow[t]{2}{*}{ C47 } & MPNST & Males & 12 & 0.003 & $3819.6(1971.4,6672.5)$ \\
\hline & & Females & 15 & 0.002 & $7788.2(4355.7,12846.2)$ \\
\hline
\end{tabular}

Abbreviation: PMR, proportionate mortality ratio.

Values in bold refer to factors significantly associated with NF1 deaths.

Even with complications such as glioma and MPNST, which would have clearly been secondary to NF1, only $29 / 48$ (60\%) had NF1 on the death certificate. For complications such as breast cancer, which have only been recently associated with NF1, ${ }^{9}$ only $1 / 9$ had NF1 recorded. The use of death certificates by Rasmussen et $^{3} \mathrm{l}^{3}$ therefore needs to be assessed against the likelihood that all deaths associated with NF1 were recorded. The 3770 deaths that recorded NF1 were from a total of 32722122 representing 1 in 8679 deaths.

The estimated birth incidence for NF1 in most reports varies between 1 in 2500 and 1 in 3000. ${ }^{10-12}$ If it is assumed that incidence and death rates remain constant, the US death certificate study is likely 
to have a substantial bias, with only $29-35 \%$ of expected NF1 deaths being recorded on the death certificate. We in common with the US study found an increased PMR from cardiovascular disease but this was only significant for males. If cardiovascular disease was more likely to be accompanied by a co-contributing cause of NF if the patient was $<30$ years this might explain the failure to detect an increased risk at all ages. Underrepresentation of NF1 deaths could also cause an exaggeration in the degree of reduction in life expectancy of NF1 patients. A second death certificate study used data from Japanese vital statistics from 1968-1992 on 605 deaths for which NF was listed as the underlying cause of death. The mean age at death in this study was 43 years. ${ }^{13}$ However, the authors did not distinguish between NF1 and NF2, and no data were available on causes of death other than NF. Additionally, because the only individuals included in the study were those who had NF1 listed as the underlying cause of death, NF was again believed to have been underascertained even by the author. ${ }^{13}$ Looking at data from the present study NF1 would only have been considered as a direct cause of death in five or six of the 50 deaths (10-12\%) after 50 years of age, but at least $48 / 80$ (60\%) of the deaths aged 50 years or less. Indeed NF1 was mentioned on the death certificate in only $12 / 50(24 \%)$ deaths $>50$ years but $34 / 79(43 \%)$ before 50 years. As such the mean age of death in the Japanese study would be expected to be biased to a younger age.

Two previous studies have suggested a higher mortality rate from malignancy in women with NF1. Although there was a higher number of female deaths in the present study due to malignancy this was not significant. Indeed the difference was accounted for, almost entirely, by the newly identified association between NF1 and breast cancer. ${ }^{9,14}$

The present study has shown typical causes of death related to NF1, but the clinician dealing with NF1 should be aware of a number of potentially preventable deaths. Quadriparesis leading to respiratory failure due to cord compression and postoperative bleeding into plexiform tumours are known complications of NF1. At least nine deaths were due to complications of radiotherapy that would not now be given for NF1-related optic gliomas. ${ }^{15,16}$ Although optic gliomas in NF1 are usually thought to be less aggressive than their sporadic counterparts ${ }^{17}$ they can occasionally be very aggressive especially in the very early onset tumours ${ }^{18}$ as seen by three deaths before 4 years of age in the present study. There was some evidence of clustering of malignancy in some families, in particular one family that has been previously reported to have had three generations affected with MPNST and rhabdomyosarcoma, ${ }^{19}$ the most recent case having a malignant gastrointestinal stromal tumour (GIST).

The current study has shown that most of the excess mortality in NF1 exists before 50 years of age and that NF1 does not clearly contribute to more than a small minority of deaths after that age. Therefore individuals living beyond 50 years without a serious NF1 complication could expect to live a near normal life expectancy. Although the current study has attempted to address most of the ascertainment biases inherent in such research the ideal study has not yet been performed. This would need to cover a cohort of NF1 patients identified with complete ascertainment and followed until every patient had died. This could be achieved with the 384 regional patients born between $1974-1993$ in our present cohort, ${ }^{12}$ but would obviously take potentially 70 years or more to mature. The 212 nationwide cohort of NF1 patients identified by Sorensen et al is close to maturity but this clearly was not a fully ascertained population.

\section{CONFLICT OF INTEREST}

The authors declare no conflict of interest.

\section{ACKNOWLEDGEMENTS}

We acknowledge the support of the NIHR Biomedical Research Centre at Central Manchester Foundation Trust.

1 Sorensen SA, Mulvihill JJ, Nielsen A: Long term follow up of von Recklinghausen neurofibromatosis: survival and malignant neoplasms. N Engl J Med 1986; 314: 1010-1015.

2 Zöller M, Rembeck B, Akesson HO, Angervall L: Life expectancy, mortality and prognostic factors in neurofibromatosis type 1. A twelve-year follow-up of an epidemiological study in Göteborg, Sweden. Acta Derm Venereol 1995; 75: 136-140.

3 Rasmussen SA, Yang Q, Friedman JM: Mortality in neurofibromatosis 1: an analysis using US death certificates. Am J Hum Genet 2001; 68: 1110-1118.

4 Evans DGR, Baser ME, McGaughran J, Sharif S, Donnelly B, Moran A: Malignant peripheral nerve sheath tumours in neurofibromatosis 1. J Med Genet 2002; 39: 311-314.

5 McGaughran JM, Harris DI, Donnai D et al: A clinical study of type 1 neurofibromatosis in North West England. J Med Genet 1999; 36: 192-196.

6 Mulvihill JJ, Parry DM, Sherman JL, Pikus A, Kaiser-Kupfer MI, Eldridge R: NIH conference. Neurofibromatosis 1 (Recklinghausen disease) and neurofibromatosis 2 (bilateral acoustic neurofibromatosis). An update. Ann Intern Med 1990; 113: 39-52.

7 Office of National Statistics: Death rates 2008. http://www.statistics.gov.uk/downloads/ theme_health/DR2008/DR_08.pdf.

8 Samuelsson B, Samuelsson S: Neurofibromatosis in Gothenburg, Sweden. I. Background, study design and epidemiology. Neurofibromatosis 1989; 2: 6-22.

9 Sharif S, Moran A, Huson SM et al: Women with neurofibromatosis 1 (NF1) are at a moderately increased risk of developing breast cancer and should be considered for early screening. J Med Genet 2007; 44: 481-484.

10 Huson SM, Compston DA, Clark P, Harper PS: A genetic study of von Recklinghausen neurofibromatosis in south east Wales. I. Prevalence, fitness, mutation rate, and effect of parental transmission on severity. J Med Genet 1989; 26: 704-711.

11 Lammert M, Friedman JM, Kluwe L, Mautner VF: Prevalence of neurofibromatosis 1 in German children at elementary school enrolment. Arch Dermatol 2005; 141: 71-74.

12 Evans DG, Howard E, Giblin C et al: Birth incidence and prevalence of tumour prone syndromes: estimates from a UK genetic family register service. Am J Med Genet 2010; 152A: 327-332.

13 Imaizumi Y: Mortality of neurofibromatosis in Japan, 1968-1992. J Dermatol 1995; 22: 191-195.

14 Walker $\mathrm{L}$, Thompson D, Easton $\mathrm{D}$ et al: A prospective study of neurofibromatosis type 1 cancer incidence in the UK. Br J Cancer 2006; 95: 233-238.

15 Evans DGR, Birch JM, Ramsden RT, Moffat D, Baser ME: Malignant transformation and new primary tumours after therapeutic radiation for benign disease: substantial risks in certain tumour-prone syndromes. J Med Genet 2006; 43: 289-294.

16 Sharif S, Ferner R, Birch J, Gattamaneni R, Gillespie J, Evans DGR: Second primary tumours in neurofibromatosis 1 (NF1) patients treated for optic glioma: substantial risks post radiotherapy. J Clin Oncol 2006; 24: 2570-2575.

17 Singhal S, Kerr B, Birch J, Lashford L, Evans DGR: Clinical characteristics of symptomatic sporadic and NF1 related optic gliomata: implications for management. Archives Dis Child 2002; 87: 65-70.

18 King A, Listernick R, Charrow J, Piersall L, Gutmann DH: Optic pathway gliomas in neurofibromatosis type 1 : the effect of presenting symptoms on outcome. Am J Med Genet A 2003; 122A: 95-99.

19 Hartley AL, Birch JM, Kelsey AM, Harris M, Jones PH: Sarcomas in three generations of a family with neurofibromatosis. Cancer Genet Cytogenet 1990; 45: 245-248. 